\title{
Robust Control Framework Based on Input-Output Models Enhanced with Uncertainty Estimation
}

\author{
Mariana RODRIGUEZ-JARA ${ }^{1}$, Hilario FLORES-MEJIA ${ }^{1}$, \\ Alejandra VELASCO-PEREZ ${ }^{2}$, Hector PUEBLA ${ }^{1 *}$ \\ ${ }^{1}$ Universidad Autónoma Metropolitana Azcapotzalco, Av. San Pablo 180, Azcapotzalco, 02200, México \\ marianarodriguez0940j@gmail.com, hpuebla@azc.uam.mx (*Corresponding author) \\ ${ }^{2}$ Universidad Veracruzana Orizaba, Orizaba, México \\ alvelasco@uv.mx
}

\begin{abstract}
A versatile and simple robust model-based control framework for regulating a class of (bio)-chemical processes is introduced. The proposed control framework departs from a simple low-order model which is enhanced by estimating model uncertainties due to model reduction, uncertain model parameters, and external disturbances. Three robust model-based control schemes are then formulated based on the enhanced simple input-output model. The proposed robust framework provides the robustness of two well-known model-based control approaches using a simple low-order model. A benchmark model of microalgae production is employed for illustrating the controller performance. Moreover, a classical PI controller and a nonlinear MPC controller are also designed and applied for comparison purposes. Numerical results show that the proposed simple robust model-based controllers are able to regulate the controlled variable to the desired reference despite external disturbances and set-point changes. Furthermore, the proposed controllers feature an acceptable performance in comparison with two of the most widely accepted controllers in the control engineering community for controlling (bio)chemical processes.
\end{abstract}

Keywords: Robust control, Model-based control, Input-output models, State observers, (Bio)-chemical processes.

\section{Introduction}

(Bio)-chemical industrial processes are procedures involving chemical and biochemical reactions in the manufacturing of items, which are useful for everyday use or serve as input for other industrial processes, usually carried out on a huge scale. The chemical process industries broadly include organic and inorganic chemical industries, the petroleum industry, and the petrochemical industry (Turton et al., 2008). Biotechnological industries comprise the food and biopharmaceutical industries (Kondakci \& Zhou, 2017; Hong et al., 2018). Moreover, biotechnological industries offer solutions to a broad spectrum of current challenges, including remediation of environmental pollution, sustainable food and energy production, and the supply of bulk chemicals (Gavrilescu \& Chisti, 2005).

Batch and fed-batch processes are widely employed in order to produce fine chemicals, pharmaceutical products, polymers, and many other materials (Hong et al., 2018). Advantages of the batch and fed-batch operation include simplicity of equipment, the ability to clean completely between batches, and years of operational experience. Nevertheless, because of their higher long-term production and better quality control, continuous processes are preferable (Calabrese \& Pissavini, 2011; Moulijn, Makkee, \& Van Diepen, 2013).
The most common operation of continuous processes for achieving the desired product quality is steady-state. However, continuous processes are very challenging to operate. Indeed, process variables are usually unknown, slow attainment of the steady-state, potential instability of operation, and the complexity of the underlying (bio)chemical reactions (Bequette, 2003; van Impe, Vanrolleghem \& Iserentant, 2013). Moreover, even in normal operational conditions, several disturbances may occur which may degrade process performance. Hence, advanced control design is of utmost relevance for keeping the system performance as close as possible to the desired one.

Most (bio)-chemical processes display nonlinear behavior due to complex interactions between transport and reaction phenomena (Bequette, 2003; van Impe, Vanrolleghem \& Iserentant, 2013). For control design purposes around a steady-state point, a linear model, obtained either by linearization or identification, is frequently used. Linear-based methods have been addressed using PID-type controllers, model predictive controllers (MPC), and $\mathrm{H} \infty$ control.

For a highly nonlinear behavior, as in the case of (bio)-chemical processes, a linear controller is inadequate for assuring stability and the closedloop performance. Indeed, neglecting system 
nonlinearities may lead to instability because of disturbances or setpoint variations (Bequette, 2003; Hangos et al., 2006).

Most advanced control algorithms rely on a process model to compute the control actions. Nonlinear model-based controllers applied in (bio)-chemical processes include Lyapunov-based methods, nonlinear $\mathrm{H} \infty$ control, sliding mode control approaches (SMC) (Bouyahia et al., 2020), nonlinear MPC (Lee \& Lee, 2014), extremum seeking control (Dewasme \& Vande Wouwer, 2020), and optimal approaches (Hangos et al., 2006; Iqbal et al., 2017). However, these approaches often rely on complicated design procedures and computationally demanding algorithms which are preventing their practical application (Saltik et al., 2018; Sariyildiz et al., 2019).

Model-based control of (bio)-chemical processes is also a challenging problem due to uncertainty related to the mathematical models of such systems. Although it is often possible to derive a phenomenological nonlinear model of the process, it can be imprecise due to uncertainties and lack of perfect knowledge (Mišković \& Hatzimanikatis, 2011; Sharifian et al., 2019). Moreover, detailed nonlinear models are impractical for control design purposes due to their high order, nonlinearities, computational demand, and model uncertainties. Thus, low-order models have been commonly used (Bequette, 2003; Smith \& Corripio, 2005).

Thus, despite industrial (bio)-chemical processes being nonlinear, many advanced control designs are based on linear models. Several authors have used linear step-response models. Internal model control (IMC) approaches use a first-order plus time delay model to design an optimal IMC (Grimholt \& Skogestad, 2018). SMC design for chemical processes uses a first-order plus time delay transfer function (Camacho \& Smith, 2000). Linear models in different regions have also been considered for an $\mathrm{H} \infty$ controller design (Galán et al., 2000). MPC uses linear identification of an input-output model and linearized approximations of the nonlinear model (Lee, 2011; Juneja \& Murthy, 2018). Recent contributions addressing models with uncertainties are as follows. In (Letchindjio et al., 2019), block-oriented systems are introduced to approximate a class of non-linear systems and design an adaptable extremum seeking approach. Bhadra et al. (2019) present a non-inferential type of nonlinear controller enhanced with an estimation scheme to compensate for the external disturbances and measurement noises. A linearized robust NMPC approach based on predicted outputs through a linear approximation of the nonlinear model was proposed by Benattia, Tebbani $\&$ Dumur (2020) to reduce the computational time of min-max robust NMPC approaches.

Departing from modeling error compensation ideas (Alvarez-Ramirez, 1999), this paper introduces a simple, systematic, and novel robust model-based control framework for a class of (bio)-chemical processes. Based on an extended input-output model with uncertainty estimation, simple, robust feedback controllers are derived. The resulting control framework allows a systematic consideration of uncertainty providing high robustness capabilities against bounded and additive uncertainties in the input-output model approximation. Moreover, simple tuning rules are proposed. A benchmark case study of microalgae production, which has been used by the control engineering community in several control studies (Bernard, 2011; Telen et al., 2017), is used for illustrating the versatility, simple design, as well as the tuning of the proposed control framework. Furthermore, to compare the performance of the proposed controllers, two of the most widely accepted control designs are also applied: a classical PI controller tuned with IMC rules and a nonlinear MPC. Numerical simulations show an acceptable closed-loop performance of the proposed controller with high robustness capabilities despite the simplicity of the controller designs.

This paper is organized as follows. Section 2 presents the extended input-output model with uncertainty estimation. In Section 3, three robust model-based controllers are derived from the extended model. In Section 4, a case study is described, and numerical studies illustrate the performance of the proposed robust feedback control framework. In Section 5, concluding remarks are provided.

\section{Simple Input-Output Model Enhanced with Model Error estimation}

For model-based control design, simple models describing the dominant input-output dynamics are highly desirable. Due to its simplicity and the capability of representing the dominant process dynamics, a first-order input-output model

https://www.sic.ici.ro 
has been commonly used for controller design purposes (Bequette, 2003; Smith and Corripio, 2005). The first-order input-output model can be obtained using different identification methods. However, since uncertainties are not considered in the input-output identification process, the inaccurate model obtained can lead to a poorly designed controller, resulting in unsatisfactory control performance.

Consider a first-order input-output model for the nominal linear dynamics given by,

$$
G_{p}(s)=\frac{Y(s)}{U(s)}=\frac{k_{p}}{\tau_{0} s+1}
$$

Where $k_{p}$ is the steady-state process gain, and $\tau_{0}$ is the process time constant. For control design purposes, the basic first-order model has proved to be the simplest one, but significant uncertainties are neglected in its formulation. Thus, the following assumption is introduced:

A1. The input-output model representation given by equation (1) is affected by unmodelled nonlinearities $\xi(y)$, with bounded variation, i.e., $|\xi(y)| \leq l_{1}|y|+l_{2}$, where $l_{1}$ and $l_{2}$ are positive constants. Moreover, external disturbances $\pi(t)$ can be either constant or persistent with bounded variation.

Then, under A1, equation (1) in the time-domain can be represented as:

$$
\frac{d y(t)}{d t}=-\tau_{0}^{-1} y(t)+k_{p} \tau_{0}^{-1} u(t)+\eta(t)
$$

where $\eta(t)=\xi(y(t))+\pi(t)$, represents model uncertainties.

Thus, the uncertainty $\eta(t)$ is seen as an unknown state. Based on the model structure given by equation (2), the uncertain state can be reconstructed from the input $u(t)$ and output $y(t)$ signals.

Hence, to account for such uncertainties for control design purposes, the model given by equation (2), enhanced with a model uncertainty estimation is considered. To this end, the following reducedorder observer is considered,

$$
\frac{d \tilde{\eta}(t)}{d t}=\tau_{e}^{-1}(\eta(t)-\tilde{\eta}(t))
$$

where $\tau_{e}$ is an estimator parameter, and $\tilde{\eta}(t)$ is the estimation of the real modeling error $\eta(t)$. The estimator in equation (3) provides an estimation of real uncertain terms using only the available output and input. After simple algebraic manipulations, the estimator can be written as follows:

$\frac{d w(t)}{d t}=\tau_{0}^{-1} y(t)-k_{p} \tau_{0}^{-1} u(t)-\tilde{\eta}(t)$

with,

$\tilde{\eta}(t)=\tau_{e}^{-1}(w(t)+y(t))$

The reduced observer in equation (4) can be considered as a signal estimator, where the modeling error signal is an extended state of the nominal model (Alvarez-Ramirez, 1999). Hence, the modeling error estimation endows the control system with robustness capabilities against model-matched uncertainties. The estimator parameter $\tau_{e}$, which determines the convergence rate of the modeling error estimation, can be selected as $\tau_{e}<0.5 \tau_{0}$.

The practical enhanced input-output model with uncertainty estimation is written then as follows,

$$
\frac{d y(t)}{d t}=-\tau_{0}^{-1} y(t)+k_{p} \tau_{0}^{-1} u(t)+\tilde{\eta}(t)
$$

which in conjunction with the estimator given by equations (4) and (5), leads to a practical and straightforward way of considering model uncertainties.

The following comments are appropriate:

- $\quad A 1$ is a realistic assumption. Indeed, according to Mišković \& Hatzimanikatis (2011) and Sharifian et al. (2019), uncertainties arise: (i) when the dynamics of actuators is not considered in the control design stage, (ii) in case of neglected high-order dynamics due to the approximation of input-output transfer function models, and (iii) in case of neglected small time-delays;

- Two main streams of robust process control for characterizing the model uncertainties are: (i) to quantify the uncertainty in terms of the bound of its gain (represented by using system norms) (Morari \& Zafiriou, 1989); (ii) to accommodate uncertainty in the process model parameters using a set of uncertainty realizations based on deterministic or stochastic approaches (Lee \& Lee, 2014). In this paper, the robustness properties are against lumped uncertain parameters in the approximated input-output model and neglected nonlinearities with bounded variations and of additive nature, which is 
the case of most nonlinear functionalities modeling (bio)-chemical processes (Bequette, 2003; Hangos et al., 2006);

- For model-based robust control designs, the proposed uncertainty framework captures external perturbations and uncertainties in a simple way, and it is mathematically tractable.

\section{Robust Control Designs Based on Enhanced Input-Output Models}

In this section, three control designs departing from the extended input-output model with uncertainty estimation are derived. First, a general control problem is stated, and some assumptions for control design purposes are introduced.

\subsection{Control Problem}

Without loss of generality, the control objective is the regulation or tracking of a desired output variable $y(t)$ to the desired reference $y_{\text {ref }}(t)$, of the class of (bio)-chemical processes modeled by equation (6) manipulating the control variable $u(t)$, despite model uncertainties and external disturbances estimated with the estimator given by equations (4) and (5).

The following additional assumptions are made:

A2. The control input $u(t)$ is subjected to saturation, i.e., $\mathrm{u}_{\min } \leq u(t) \leq \mathrm{u}_{\max }$.

$A 3$. The output variable $y(t)$ is measured continuously without measurement delay.

$A 4$. The desired reference $y_{\text {ref }}(t)$ and its time derivative are available and bounded.

The following two observations can be made:

- For practical implementation purposes, the control input $u(t)$, which in several (bio)chemical processes is restricted to different flow rates, is physically limited;

- Assumption A3 is realistic. Indeed, main on-line and instantaneously measurable variables in (bio)-chemical processes include temperature, $\mathrm{pH}$, conductivity, flow rates, and chemical compositions. Moreover, even in the absence of an on-line measurement of the desired controlled variable $y(t)$, a state estimator design can be considered (Hangos et al., 2006).

\subsection{Simple Robust Model-based Control}

Consider a desired first-order asymptotically closed-loop behavior. The following inverse feedback function for the control input is proposed (Alvarez-Ramirez, 1999),

$u(t)=\frac{\tau_{0}}{k_{p}}\left\{\left(\frac{1}{\tau_{0}}-\frac{1}{\tau_{c}}\right) e(t)+\frac{d e(t)}{d t}-\tilde{\eta}(t)\right\}$

where $\tau_{c}>0$ is a controller parameter. Under the proposed controller, a first-order dynamics of the regulation error is obtained, such that asymptotic convergence $e(t) \rightarrow 0$, and so $y(t) \rightarrow y_{\text {ref }}(t)$ is guaranteed.

Tuning of parameter $\tau_{c}$ can be made to attain a satisfactory closed-loop response following the simple rule (Alvarez-Ramirez, 1999): $\tau_{e}<\tau_{c}<\tau_{0}$.

\subsection{Extended Sliding Mode Control}

Sliding mode control is a powerful, robust control method suitable for dealing with model nonlinearity and parameter uncertainties. The control design is derived in two-steps (Slotine \& Li, 1991):

1. Sliding phase: Design a sliding surface $s(t)$, such that $s(t)=0$ and $d s(t) / d t=0$, along which the controlled variable slides to its desired value. A continuous control action is obtained;

2. Reaching phase: Design a discontinuous control action that forces the controlled state to the sliding surface when $s(t) \neq 0$.

Several modifications have been introduced and applied in (bio)-chemical processes to improve the robustness capabilities against structured uncertainties and reduce chattering effects (Kanthalakshmi \& Annal, 2018; Utkin et al., 2020).

Departing from the ideas introduced by Slotine and $\mathrm{Li}$ (1991), the sliding surface is proposed as,

$s(t)=e(t)+\lambda \int_{0}^{t} e(\tau) d \tau$

Based on a first-order input-output model, AbuRmileh et al. (2010) introduce a simple controller, which is extended with the proposed control framework as follows,

$u(t)=\frac{\tau_{0}}{k_{p}}\left(-\lambda e(t)+\frac{1}{\tau_{0}} y(t)-\tilde{\eta}(t)\right)+k_{D} \operatorname{sign}(s(t))$ 
where $k_{D}$ and $\lambda$ are controller parameters, which, based on the input-output model, are proposed as $k_{D}=0.1 / k_{p}$ and $\lambda=0.5 / \tau_{0}$ (Abu-Rmileh et al., 2010).

\subsection{Extended Generic Model Control}

The standard Generic Model Control (GMC) consists in matching PI control actions with a nonlinear process model (Lee \& Sullivan, 1988). Using concepts from differential geometry and a Luenberger-type nonlinear state estimator, an extended GMC controller was introduced by Jana (2013).

Consider the extended dynamic simple model given by equation (6) with the reference system given by,

$$
\frac{d y_{r e f}(t)}{d t}=K_{1} e(t)+K_{2} \int_{0}^{t} e(\tau) d \tau
$$

The extended GMC is formulated as the standard $\mathrm{GMC}$, i.e., the dynamics of the process output given by equation (6), follows the desired reference system given by equation (10). Thus,

$-\frac{1}{\tau_{0}} y(t)+\frac{k_{p}}{\tau_{0}} u(t)+\tilde{\eta}(t)=K_{1} e(t)+K_{2} \int_{0}^{t} e(\tau) d \tau$

and solving $u(t)$ yields,

$$
u(t)=\frac{\tau_{0}}{k_{p}}\left\{\frac{1}{\tau_{0}} y(t)-\tilde{\eta}(t)+K_{1} e(t)+K_{2} \int_{0}^{t} e(\tau) d \tau\right\}
$$

Two approaches for calculating controller parameters $K_{1}$ and $K_{2}$ are as follows (Lee \& Sullivan, 1988).

The first approach is by using the following formulas,

$$
K_{1}=2 \tau_{c}^{-1} \xi, \quad K_{2}=\tau_{c}^{-2}
$$

where the parameters $\xi$ and $\tau_{c}$ can be set in two steps: (i) determine to give the desired shape of the closed-loop response, and (ii) choose $\tau_{c}$ to modulate the timing of the closed-loop response concerning the open-loop rate of response $\tau_{0}$.

The second approach is to examine the characteristic equation of the closed-loop system, given by,

$s^{2}+K_{1} s+K_{2}$

and set the location of the two closed-loop poles to solve equation (14) for $K_{1}$ and $K_{2}$.

\subsection{Extension to a Second-orden Input- Output Model}

In the preceding sections, the simple robust control framework was formulated for a first-order inputoutput model. Consider a second-order system given as,

$G_{p}(s)=\frac{Y(s)}{U(s)}=\frac{k_{p}}{\tau_{0}^{2} s^{2}+2 \varepsilon \tau_{0} s+1}$

where $\varepsilon$ is a damping coefficient. Second-order input-output models can describe several (bio)chemical processes, including first-order timedelayed input-output models with a first-order Taylor series approximation to the time-delay term (Camacho \& Smith, 2000; Bequette, 2003).

In the time-domain, the second-order uncertain model can be written as,

$\frac{d y(t)}{d t}=z(t)$

$\frac{d z(t)}{d t}=-\frac{2 \varepsilon}{\tau_{0}} z(t)-\frac{1}{\tau_{0}^{2}} y(t)+\frac{k_{p}}{\tau_{0}^{2}} u(t)+\eta(t)$

In this case, the simultaneous estimation of $z(t)$ and the lumped uncertainties $\eta(t)$, from the available output $y(t)$ are given as,

$$
\begin{aligned}
& \frac{d w_{1}(t)}{d t}=-\frac{2 \varepsilon}{\tau_{0}} \tilde{z}(t)-\frac{1}{\tau_{0}^{2}} y(t)+\frac{k_{p}}{\tau_{0}{ }^{2}} u(t)+\tilde{\eta}(t)-\frac{2}{\tau_{e}} \tilde{z}(t) \\
& \frac{d w_{2}(t)}{d t}=-\frac{1}{\tau_{e}^{2}} \tilde{z}(t)
\end{aligned}
$$

with,

$$
\begin{aligned}
& \tilde{\eta}(t)=w_{2}(t)+\tau_{e}^{-2} y(t) \\
& \tilde{z}(t)=w_{1}(t)+2 \tau_{e}^{-1} y(t)
\end{aligned}
$$

Hence, previous control designs can be formulated using a second-order model with uncertainty estimation.

\subsection{Robustness and Stability Issues}

Although formal robustness and stability analysis are beyond the scope of this paper, the closedloop system can be analyzed using stability results from singular perturbation systems (Khalil, 1992), where the dynamics of the estimation error, $e_{e}(t)=\eta(t)-\tilde{\eta}(t)$, is the fast dynamics. Moreover, its reduced model is the closed-loop system resulting from the base version of the extended 
control designs formulated in this paper. Finally, robustness properties of the closed-loop system are provided by the estimation and cancelation of uncertain terms in each control design.

\subsection{Nonlinear MPC}

For comparison purposes, the standard NMPC is considered. NMPC is a powerful control method for controlling nonlinear, constrained, and uncertain chemical and biological processes (Lee, 2011; Piceno-Díaz et al., 2020).

The objective of an NMPC formulation is to find the best control sequence (until a given control horizon, $M$ ) such that a given cost function is minimized. The corresponding optimization problem relies on predicting future behavior in a given prediction horizon $(N)$.

The standard NMPC can be formulated as follows (Lee \& Lee, 2014):

$$
\min _{u_{k}} J=\gamma_{1} \sum_{j=0}^{N}\left\|y_{k+j}-y_{r e f}\right\|^{2}+\gamma_{2} \sum_{j=0}^{M}\left\|\Delta u_{k+j}\right\|^{2}
$$

subject to,

$x_{k+1}=f\left(x_{k}, u_{k}, \tilde{\theta}\right)$

$h\left(x_{k}, u_{k}, \tilde{\theta}\right) \leq 0$

$x_{\min } \leq x_{k} \leq x_{\max }$

$u_{\min } \leq u_{k} \leq u_{\max }$

where $y_{k+j}$ is the predicted value of the controlled variable using the non-linear process model, $y_{\text {ref }}$ is the desired reference, $u_{k}$ denotes the sequence of control inputs and $\Delta u_{k+j}$ is the control move at instant $k+j$, i.e., $u_{k+j}-u_{k+j-1} \cdot \gamma_{1}$ and $\gamma_{2}$ are weighting factors used to set the ratio of importance between costs due to deviation from desired states and costs due to control moves. Nominal parameter values are denoted as $\tilde{\theta}$.It can also be mentioned that function $f$ in (21) represents the discretized nonlinear dynamical model of the system and $h$ is the set of process system constraints. Moreover, $k$ is the sampling instant, which relates to processing time $t$ via $t=k T_{s}$, where $T_{s}$ is the sampling time. Controller parameters are the prediction horizon $N$, the control horizon $M$, the sampling time $T_{s}$, and the weighting factors $\gamma_{1}$ and $\gamma_{2}$.

The standard NMPC algorithm has acceptable robustness capabilities. Indeed, the correction of the model's future prediction and the actual system's response in each iterative step of the
NMPC algorithm alleviates slight model mismatch providing some degree of robustness.

The simultaneous full-discretization approach is pursued to implement the NMPC algorithm, leading to a nonlinear programming (NLP) problem at each time interval (Biegler, 2007). To this end, both the state and control profiles are discretized by means of orthogonal collocation on finite elements using Legendre polynomials with three internal collocation points (Finlayson, 1980). The resulting NLP optimization problem was programmed in Matlab using the fmincon function with the interior point optimization method.

\section{Numerical Example}

In this section, the derived robust model-based control schemes in the above section are evaluated using a benchmark case study of microalgae production. First, the case study is described. Then, the optimal steady-state condition is computed. Finally, the robust model-based control schemes are applied and evaluated under external disturbances and set-point changes. Furthermore, a comparison with a classical PI controller and an NMPC controller is presented.

\subsection{Microalage Growth}

The recently renewed interest in microalgae production stems from its capacity to accumulate large amounts of carbohydrates and neutral lipids, which can be used as a feedstock for biorefineries (Li et al., 2008).

Consider a biological chemostat of volume $V$, made of a transparent material. The source of light for the microalgae growth is provided via external lamps surrounding the chemostat. The input stream with a volumetric flow rate $(F)$ contains the input organic substrate $\left(S_{i n}\right)$. The output stream comprises the non-consumed substrate $(S)$ and the produced microalgae biomass $(X)$.

A simple non-structured, non-segregated model of three main species is considered for the continuous microalgae production in the chemostat (Bernard, 2011).

Model assumptions are (Droop, 1983; Darvehei, Bahri \& Moheimani, 2018): (i) Homogeneous conditions. (ii) Constant volume, external light intensity, and temperature. (iii) The organic load is lumped in a single kind of substrate, $S$. (iv) 
The microalgae biomass is lumped in a single state, $X$. (v) the kinetics of the microalgae growth is described with the Droop model, which considers an internal substrate (nutrients) pool within the cells, denoted as the internal quota $Q_{N^{*}}$

The mathematical model is given by:

$$
\begin{aligned}
& \frac{d X(t)}{d t}=-D(t) X(t)+\bar{\mu}\left(1-\frac{K_{Q}}{Q_{N}(t)}\right) X(t) \\
& \frac{d Q_{N}(t)}{d t}=\rho_{m} \frac{S(t)}{k_{s}+S(t)}-\bar{\mu}\left(Q_{N}(t)-K_{Q}\right) \\
& \frac{d S(t)}{d t}=D(t)\left(S_{i n}-S(t)\right)-\rho_{m} \frac{S(t)}{k_{s}+S(t)} X(t)
\end{aligned}
$$

where $S(t)(\mu \mathrm{mol} / \mathrm{L}), X(t)\left(\mu \mathrm{m}^{3} / \mathrm{L}\right)$, and $Q_{N}(t)$ $\left(\mu \mathrm{mol} / \mu \mathrm{m}^{3}\right)$ represent the concentrations of substrate, microalgae biomass, and the internal quota, respectively. The input substrate is $S_{\text {in }}(\mu \mathrm{mol} / \mathrm{L})$. The dilution rate is $D(t)=F(t) / V\left(\right.$ day $\left.^{-1}\right)$. Droop kinetic parameters are the maximum kinetic growths $\rho_{m}\left(\mu \mathrm{mol} / \mu \mathrm{m}^{3}\right.$ day $)$ and $\bar{\mu}\left(\right.$ day $\left.^{-1}\right)$ for the substrate consumption and the internal quota, respectively. $K_{Q}\left(\mu \mathrm{mol} / \mu \mathrm{m}^{3}\right)$ is the minimum growth constant for biomass growth. $k_{s}(\mu \mathrm{mol} / \mathrm{L})$ is the saturation constant for the substrate consumption.

The nominal or base operation is simulated with the parameter values given by $S_{i n}=100$ $\mu \mathrm{mol} / \mathrm{L}, k_{s}=0.105 \mu \mathrm{mol} / \mathrm{L}, K_{Q}=1.8 \mu \mathrm{mol} / \mu \mathrm{m}^{3}$, $\mu=21 /$ day, $\rho_{m}=9.3 \mu \mathrm{mol} / \mu \mathrm{m}^{3}$ day, and $D=1.3$ day $^{-1}$. A single steady-state which is given by $\left[S, Q_{N}, X\right]^{*}=\left[0.268 \mu \mathrm{mol} / \mathrm{L}, 5.142 \mu \mathrm{mol} / \mu \mathrm{m}^{3}\right.$, $\left.19.392 \mu \mathrm{m}^{3} / \mathrm{L}\right]$ is obtained with the above parameter values. Moreover, standard linear stability analysis of the equilibrium point was performed to corroborate the stable behavior.

Figure 1 shows a step-change in the dilution rate to derive the input-output first-order model. Note that a smooth response is obtained. Based on the input-output response the first-order parameters $k_{p}=-31.7$ and $\tau_{0}=2$ are obtained.

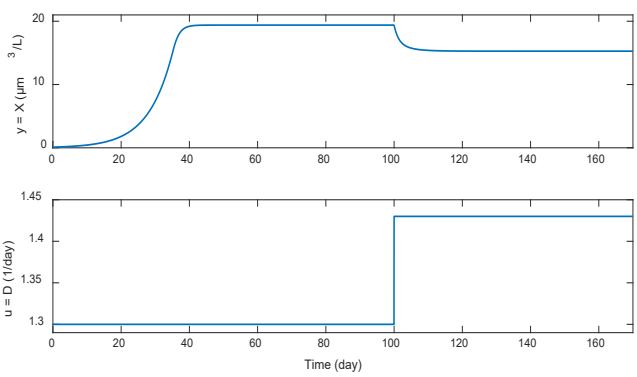

Figure 1. Step identification of the Droop model

\subsection{Control Problem}

The control problem is set as the regulation of the microalgae biomass concentration $y(t)=X(t)$, at the optimal productivity condition, using as the manipulated variable the dilution rate, $u(t)=D(t)$.

Linear and nonlinear MPC approaches were applied to similar control problems for optimal microalgae growth using the Droop model (Benattia, Tebbani \& Dumur, 2020) and a simple three-state experimentally validated model (Juneja \& Murthy, 2018).

The optimal conditions are obtained by solving the following nonlinear optimization problem:

$\min J=-D X$

subject to,

$$
\begin{aligned}
& 0=-D X+\bar{\mu}\left(1-\frac{K_{Q}}{Q_{N}}\right) X \\
& 0=\rho_{m} \frac{S}{k_{s}+S}-\bar{\mu}\left(Q_{N}-K_{Q}\right) \\
& 0=D\left(S_{i n}-S\right)-\rho_{m} \frac{S}{k_{s}+S} X
\end{aligned}
$$

$S \leq S_{\text {in }}$

$D_{\text {min }} \leq D \leq D_{\text {max }}$

Constraints of the optimization problem correspond to the steady-state model equations (24), the upper limit of the input substrate given by equation (25), and minimum and maximum constraints on the decision variable given by equation (26), with $D_{\min }=0.1$ day $^{-1}$, and $D_{\max }=$ 5 day $^{-1}$.

The resulting optimization problem is nonlinear and constrained. In this case, the interior-point algorithm available in Matlab for solving the NLP optimization problem was applied. The resulting optimal steady-state is given by $\left[S, Q_{N}, X, D\right]$ op $=\left[3.59 \mu \mathrm{mol} / \mathrm{L}, 0.066 \mu \mathrm{mol} / \mu^{3}{ }^{3}, 27.8 \mu \mathrm{m}^{3} / \mathrm{L}, 1\right.$ $1 /$ day], which correspond to a $10 \%$ increase in the nominal biomass microalgae productivity.

The control action is activated at $t=100$ days. An external perturbation on the input substrate is applied at $t=200$ days. Finally, a set-point change is applied at $t=300$ days. 


\subsection{Simple Robuts Control Design}

Figure 2 shows the closed-loop behavior using the simple robust model-based control design and three sets of controller parameters chosen according to the tuning guidelines described in the section above. The comparison with a PI controller with IMC-PI tuning parameters is also illustrated.

Figure 2 shows that the proposed robust modelbased controller can regulate the output despite the presence of the input substrate disturbance. It can also be seen that the change of set point is achieved with an acceptable control effort. The best closed-loop performance is obtained for the first set of controller parameters, $\tau_{e}=1.0, \tau_{c}=1.5$.
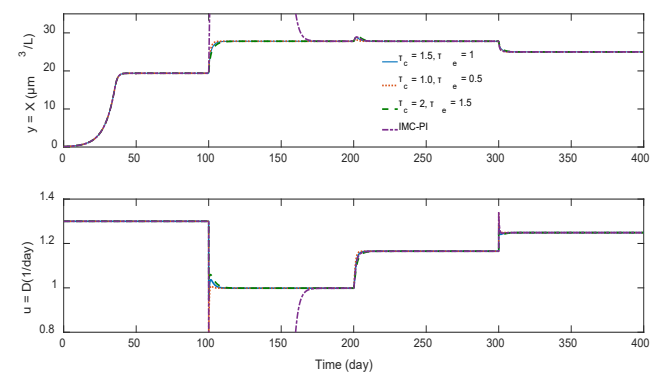

Figure 2. Closed-loop performance of the simple robust model-based controller for the Droop model

Lower values of the controller parameters lead to a more aggressive control action. On the other hand, higher values for controller parameters lead to a smoother but also slower closed-loop response. However, as it is shown in Figure 2, the proposed simple robust model-based controllers display a superior performance to that of the PI for the control input saturation at its lower value when the controller is activated at 100 days.

\subsection{Extended Sliding Mode Control}

The closed-loop performance of the extended SMC approach is shown in Figure 3.
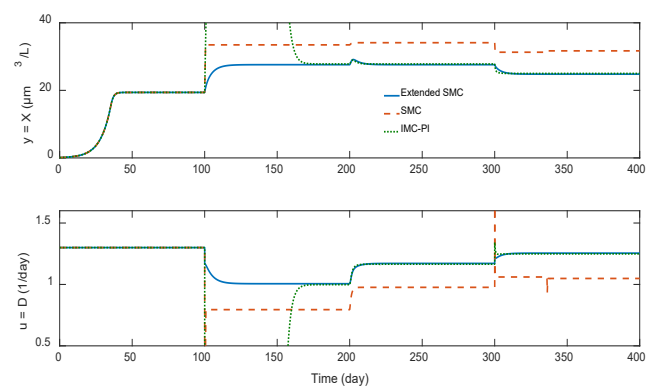

Figure 3. Closed-loop performance of the extended SMC for the Droop model

It can be noticed that the proposed extended $\mathrm{SMC}$ is able to regulate the microalgae biomass concentration with acceptable closed-loop behavior. Moreover, the original SMC approach is unable to achieve the desired reference. It is to be noted that for the original SMC approach, the tuning parameters were performed using a trialand-error procedure instead of the tuning rules described in the above section. However, for the extended SMC approach that rules were applied leading to the illustrated closed-loop behavior.

\subsection{Extended Generic Model Control}

The extended GMC was implemented using three sets of parameter values of $K_{1}$ and $K_{2}$ with the pole placement approach.

Figure 4 illustrates the numerical simulations of the closed-loop behavior of the extended GMC. It is to be noted that the closed-loop performance is similar to that of PI controller.
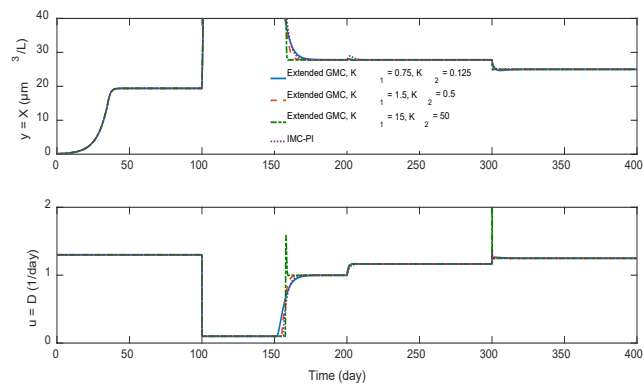

Figure 4. Closed-loop performance of the extended GMC for the Droop model

The poor performance of both controller approaches can be related to the effect of the integral action producing an initial aggressive control action that saturates the control input to its lower limit. However, after this transitory dynamics, and when the disturbances are applied along with the set-point change, the closed-loop performance displays an acceptable behavior.

\subsection{NMPC}

Figure 5 shows the numerical results of the NMPC implementation and the comparison with the simple robust model-based controller and the extended SMC. NMPC parameters are $\left[\Delta t, T_{s}, N, M, \gamma_{1}, \gamma_{2}\right]=[1,0.5,2,1,10,1]$.

It can be noticed that the NMPC approach displays the best closed-loop behavior with more pronounced peaks at the activation of the control action and the setpoint change. The NMPC approach is also able to reject the external perturbation on the input substrate. However, despite the simplicity of its 
implementation the proposed robust model-based controller and the extended SMC show similar closed-loop performances. Moreover, it is to be noted that the NMPC formulation uses the nonlinear model for the control design and requires the full state measurement for implementation purposes in the current applied formulation.
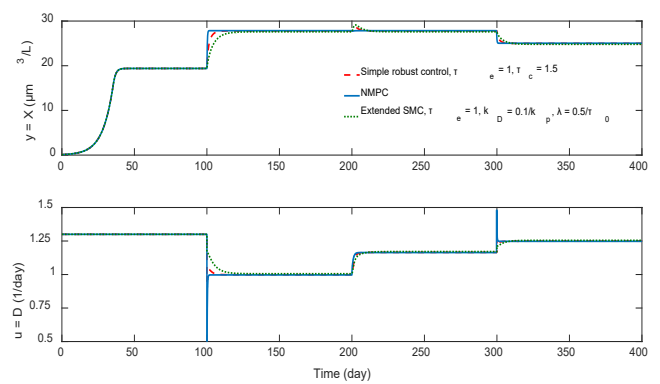

Figure 5. Closed-loop performance of the NMPC for the Droop model

\section{Conclusion}

This paper presents a simple robust control framework for regulating a class of (bio)-chemical

\section{REFERENCES}

Abu-Rmileh A., Garcia-Gabin, W. \& Zambrano, D. (2010). A Robust Sliding Mode Controller with Internal Model for Closed-Loop Artificial Pancreas, Medical \& Biological Engineering \& Computing, 48(12), 1191-1201.

Alvarez-Ramirez, J. (1999). Adaptive Control of Feedback Linearizable Systems: a Modelling Error Compensation Approach. International Journal of Robust and Nonlinear Control, 9(6), 361-377.

Benattia, S.E., Tebbani, S. \& Dumur, D. (2020) Linearized min-max Robust Model Predictive Control: Application to the Control of a Bioprocess, International Journal of Robust and Nonlinear Control, 30(1), 100-120.

Bequette, B.W. (2003). Process Control: Modeling, Design, and Simulation. Prentice Hall Professional.

Bernard, O. (2011). Hurdles and Challenges for Modelling and Control of Microalgae for $\mathrm{CO} 2$ Mitigation and Biofuel Production, Journal of Process Control, 21(10), 1378-1389.

Bhadra, S., Panda, A., Bhowmick, P., Goswami, S. \& Panda, R. C. (2019). Design and Application of Nonlinear Model-Based Tracking Control Schemes Employing DEKF Estimation, Optimal Control Applications \& Methods, 40(5), 938-960.

Biegler, L.T. (2007). An Overview of Simultaneous Strategies for Dynamic Optimization, Chemical processes. The robust control framework is formulated in three steps: (i) step identification of a low first-order model, (ii) derivation of an enhanced first-order model, with the estimation of model uncertainties associated with the model reduction and external disturbances, and (iii) the design of robust control schemes based on the enhanced model. The resulting controllers have a simple structure with simple tuning rules based on the process time constant. The proposed robust control framework is applied to a benchmark case study of microalgae biomass production. The effectiveness of the proposed, approach is demonstrated with the derivation of three robust control designs with a good closedloop performance despite external perturbations. Moreover, the comparison with a NMPC design shows similar closed-loop performance without the requirements of a full state measurement and a bigger computational effort.

Engineering and Processing: Process Intensification, 46(11), 1043-1053.

Bouyahia, S., Semcheddine, S., Talbi, B., Boutalbi, O. \& Terchi, Y. (2020). An Adaptive Super-Twisting Sliding Mode Algorithm for Robust Control of a Biotechnological Process, International Journal of Dynamics and Control, 8, 581-591.

Calabrese, G. S. \& Pissavini, S. (2011). From Batch to Continuous Flow Processing in Chemicals Manufacturing, AIChE Journal, 57(4), 828-834.

Camacho, O. \& Smith, C.A. (2000). Sliding Mode Control: an Approach to Regulate Nonlinear Chemical Processes, ISA Transactions, 39(2), 205-218.

Darvehei, P., Bahri, P. A. \& Moheimani, N. R. (2018). Model Development for the Growth of Microalgae: A Review, Renewable \& Sustainable Energy Reviews, 97, 233-258.

Dewasme, L. \& Vande Wouwer, A. (2020). Model- Free Extremum Seeking Control of Bioprocesses: A Review with a Worked Example, Processes, 8(10), 1209.

Droop, M.R. (1983). 25 Years of Algal Growth Kinetics a Personal View, Botanica Marina, 26(3), 99-112.

Finlayson, B. A. (1980). Nonlinear Analysis in Chemical Engineering. McGraw-Hill: Chemical Engineering Series. 
Galán, O., Romagnoli, J.A. \& Palazoglu, A. (2000). Robust $\mathrm{H} \infty$ Control of Nonlinear Plants Based on Multi-Linear Models: An Application to a Bench-Scale $\mathrm{pH}$ Neutralization Reactor, Chemical Engineering Science, 55 (20), 4435-4450.

Gavrilescu, M. \& Chisti, Y. (2005). Biotechnology-A Sustainable Alternative for Chemical Industry, Biotechnology Advances, 23(7-8), 471-499.

Grimholt, C. \& Skogestad, S. (2018). Optimal PI and PID Control of First-Order Plus Delay Processes and Evaluation of the Original and Improved SIMC Rules, Journal of Process Control, 70, 36-46.

Hangos, K.M., Bokor, J. \& Szederkényi, G. (2006). Analysis and Control of Nonlinear Process Systems, Springer Science \& Business Media.

Hong, M.S., Severson, K.A., Jiang, M., Lu, A.E., Love, J.C. \& Braatz, R.D. (2018). Challenges and Opportunities in Biopharmaceutical Manufacturing Control, Computers \& Chemical Engineering, 110, 106-114.

Iqbal, J., Ullah, M., Khan, S.G., Khelifa, B. \& Ćuković, S. (2017). Nonlinear Control Systems-A Brief Overview of Historical and Recent Advances, Nonlinear Engineering, 6 (4), 301-312.

Jana, A.K. (2013). Differential Geometry-Based Adaptive Nonlinear Control Law: Application to an Industrial Refinery Process, IEEE Transactions on Industrial Informatics, 9(4), 2014-2022.

Juneja, A. \& Murthy, G.S. (2018). Model Predictive Control Coupled with Economic and Environmental Constraints for Optimum Algal Production, Bioresource Technology, 250, 556-563.

Khalil, H.K. (1992). Nonlinear Systems, Macmillan, New York.

Kanthalakshmi, S. \& Annal, W.P. (2018). Real Time Implementation of Adaptive Sliding Mode Controller for a Nonlinear System, Studies in Informatics and Control, 27(4), 395-402. DOI: 10.24846/ v27i4y201803

Kondakci, T. \& Zhou, W. (2017). Recent Applications of Advanced Control Techniques in Food Industry, Food and Bioprocess Technology, 10(3), 522-542.

Lee, P. L. \& Sullivan, G. R. (1988). Generic model control (GMC). Computers and Chemical Engineering, 12(6), 573-580.

Lee, J. H. (2011). Model Predictive Control: Review of the Three Decades of Development, International Journal of Control, Automation and Systems, 9, 415.

Lee, J. H. \& Lee, J. M. (2014). Progress and Challenges in Control of Chemical Processes, Annual Review of Chemical and Biomolecular Engineering, 5, 383-404.

Letchindjio, C.F., Dewasme, L., Deschênes, J. S. \& Wouwer, A. V. (2019). An Extremum Seeking Strategy Based on Block-Oriented Models: Application to
Biomass Productivity Maximization in Microalgae Cultures, Industrial \& Engineering Chemistry Research, 58(30), 13481-13494.

Li, Y., Horsman, M., Wu, N., Lan, C. Q. \& DuboisCalero, N. (2008). Biofuels from Microalgae, Biotechnology Progress, 24(4), 815-820.

Mišković, L. \& Hatzimanikatis, V. (2011). Modeling of Uncertainties in Biochemical Reactions, Biotechnology and Bioengineering, 108(2), 413-423.

Morari, M. \& Zafiriou, E. (1989). Robust Process Control. Englewood-Cliffs.

Moulijn, J.A., Makkee, M. \& Van Diepen, A. E. (2013). Chemical Process Technology. John Wiley \& Sons.

Piceno-Díaz, E.R., Ricardez-Sandoval, L.A., Gutierrez- Limon, M.A., Méndez-Acosta, H.O. \& Puebla, H. (2020). Robust Nonlinear Model Predictive Control for Two-Stage Anaerobic Digesters, Industrial \& Engineering Chemistry Research, 59(52), 22559-22572.

Saltık, M.B., Özkan, L., Ludlage, J.H., Weiland, S. \& Van Den Hof, P.M. (2018). An Outlook on Robust Model Predictive Control Algorithms: Reflections on Performance and Computational Aspects, Journal of Process Control, 61, 77-102.

Sariyildiz, E., Oboe, R. \& Ohnishi, K. (2019). Disturbance Observer-Based Robust Control and Its Applications: 35th Anniversary Overview, IEEE Transactions on Industrial Electronics, 67(3), 2042-2053.

Sharifian, S., Sotudeh-Gharebagh, R., Zarghami, R., Tanguy, P. \& Mostoufi, N. (2019). Uncertainty in Chemical Process Systems Engineering: A Critical Review, Reviews in Chemical Engineering, (Aheadof-Print).

Slotine, J. J. E. \& Li, W. (1991). Applied Nonlinear Control. Prentice Hall.

Smith, C. A. \& Corripio, A. B. (2005). Principles and Practices of Automatic Process Control. John Wiley \& Sons.

Telen, D., Houska, B., Vallerio, M., Logist, F. \& Van Impe, J. (2017). A Study of Integrated Experiment Design for NMPC Applied to the Droop Model, Chemical Engineering Science, 160, 370-383.

Turton, R., Bailie, R.C., Whiting, W.B. \& Shaeiwitz, J.A. (2008). Analysis, Synthesis and Design of Chemical Processes. Pearson Education.

Utkin, V., Poznyak, A., Orlov, Y. \& Polyakov, A. (2020). Conventional and High Order Sliding Mode Control, Journal of the Franklin Institute, 357(15), 10244-10261.

Van Impe, J.F., Vanrolleghem, P.A. \& Iserentant, D.M. (Eds.). (2013). Advanced Instrumentation, Data Interpretation, and Control of Biotechnological Processes. Springer Science \& Business Media. 